\title{
Gender differences in subjective discontinuation symptoms associated with ketamine use
}

\author{
Wen-Yin Chen ${ }^{1}$, Ming-Chyi Huang ${ }^{1,2}$ and Shih-Ku Lin ${ }^{1,2^{*}}$
}

\begin{abstract}
Background: Recent substance abuse research indicates gender differences in the substance-related epidemiology, biological responses, progression to dependence, medical consequences and treatments. Studies exploring human sex-different responses to ketamine are rare and there has been no systemic survey of gender differences in ketamine use. Determining whether females are more susceptible than males to ketamine withdrawal symptoms and adverse effects is important, because it associated with treatment retention and outcome in drug users.
\end{abstract}

Methods: The Taiwanese juridical system has implemented a new regulation on ketamine in the year 2009. Ketamine users who are caught by the police, are mandated to attend an educational program. We recruited ketamine offenders from February 2010 to May 2012 at the Kunming branch of the Taipei City Hospital, where the educational classes are held. A designed questionnaire was performed to gather information about demographic characteristics, discontinuation symptoms, concomitant use of other substances, and subjective experience of memory impairment or urinary discomforts, and to compare the gender differences.

Results: A total of 1,614 ketamine users were surveyed and most of them were males (83.8\%), with an average age of $26.3 \pm 5.4$ years. Female ketamine users presented significantly more discontinuation symptoms such as anxiety, dysphoria, and tremors compared with male users. $72.4 \%$ of total ketamine users smoked cigarettes concomitantly. Male ketamine users had a higher rate of concomitant betel nut use, while female ketamine users had a higher rate of concomitant hypnotic and alcohol use. $76 \%$ of total ketamine users reported cognitive impairment and 51.6\% mentioned urinary symptoms. Furthermore, female ketamine users self-reported significantly greater levels of severity in cognitive impairment and urinary discomforts compared with male users. Less than $10 \%$ of total ketamine users in our study reported the desire to transfer for medical intervention or treatment, despite the high rates of discontinuation symptoms and negative physical side effects.

Conclusions: Gender differences were noted in the subjective experience of discontinuation symptoms, concomitant substance use, and severity of impairment related to ketamine use. However, the probable cause of the gender differences found in this study requires further investigation. We hoped our study will stimulate further research in this field.

Keywords: Ketamine, Epidemiology, Discontinuance symptoms, Gender difference

\footnotetext{
* Correspondence: sklin@tpech.gov.tw

'Department of Addiction Science, Taipei City Hospital and Psychiatric Center, 309 Songde Road, Xinyi District, Taipei 110, Taiwan

${ }^{2}$ Department of Psychiatry, College of Medicine, Taipei Medical University,

Taipei, Taiwan
} 


\section{Background}

Ketamine is an anesthetic derivative of phencyclidine (PCP) with dissociative, analgesic and psychedelic properties. Although ketamine is classified as a hallucinogen, it is widely used in a variety of anesthetic procedures in pediatric, obstetric and geriatric patients [1]. Moreover, the antidepressant effects of ketamine in treating refractory depression were reported in recent years [2].

According to the latest systemic review by Morgan and Curran [3], there are now increasing concerns about the harmful physical and psychological consequences of repeated misuse of ketamine. Acute physical harms include risk of death from accidents, acute cardiac risk from poisoning; ketamine-induced chronic physical harms include ulcerative cystitis, renal dysfunction and 'k-cramps'. Frequent, daily use is also associated with neurocognitive impairment, and most robustly, deficits in working and episodic memory [4]. Psychological effects of chronic ketamine use were related to depression and psychosis [5]. Ketamine withdrawal symptoms characterized by anxiety, shaking, sweating, palpitations and carvings seem to be key problems in frequent ketamine users and have been published by many case studies [6-8]. However, there has yet to be a coherent and agreed upon description of a specific ketamine withdrawal syndrome to guide treatment and diagnosis. A rapid development of tolerance was demonstrated in ketamine users, and many frequent users are concerned about addiction and report trying but failing to stop using ketamine. Ketamine dependence can lead to costly health services, which include the treatment of the addiction and its related impairments in social functions, as well as the chronic physical health problems that will likely incur follow-up costs across the lifespan.

Recreational use of ketamine was first documented on the west coast of the United States in the early 1970s [9]. Ketamine has not been a major drug of abuse within the past two decades, and only a few cases have been reported during this time $[10,11]$. After the ecstasy epidemic, ketamine use has become more popular in recent years around the world, especially as a common club drug among the dance, rave, and squat party scenes. It is also becoming popular in the United Kingdom [12,13], Australia [14], Hong Kong [15,16], and Mainland China [17]. In the latest World Drug Report by United Nations Office on Drugs Control (UNODC) 2013 [18], Ketamine belong to new psychoactive substance (NPS) and not controlled by international drug conventions, but which may pose a public health threat. Part of ketamine's growing popularity in South-East Asia may be due to its lower status in the regulatory systems and lower price point as a substitute for the increasingly expensive 'ecstasy' or methamphetamine.

Ketamine has also become a commonly abused substance in Taiwan during recent years, especially among youths and adolescents [19-21]. Evidence showed that most ketamine users in Taiwan also used ecstasy (MDMA) concomitantly. Detected rates of MDMA and ketamine were found to be as high as $76 \%$ and $47 \%$ respectively among rave party participants in Taiwan [20]. Leung et al. [22] conducted a focus group with club drug users and noted that there was a special drug use sequence widely practiced by Taiwanese poly-drug users. In a single drug use episode, MDMA was usually the first drug used, followed by ketamine and then marijuana. This unique sequence of polydrug use in a single episode is called "Trinity".

Ketamine is scheduled as level III in the "Narcotic Harm Control Act" in Taiwan. Pure ketamine use is regarded as an offense and not a crime. One reason for the increase in the abuse of ketamine in recent years is the lack of restriction by authorities. Reports from the Ministry of Justice showed increase in both the amount of street ketamine seized by the police, as well as the number of offenders (namely drug dealers or traffickers). Around 3000 to 4000 offenders were caught by the police in Taiwan per month during the year 2012.

Recent substance abuse research indicates gender differences in the substance-related epidemiology, social factors, biological responses, progression to dependence, medical consequences, co-occurring psychiatric disorders, and barriers to treatment entry, retention, and completion [23]. For examples, in alcohol drinking, women's drinkingrelated problems appear to progress more quickly than those of men [24], and women report more severe problems and experience more health-related consequences from substance use [25]. Stimulants may enhance sex differences in the dopamine system in limbic regions, which may partly mediate sex difference in drug abuse [26]. Craving and relapse of drug seeking in abstinent individuals have also been found to differ between men and women, and there is robust evidence that women are more likely to initiate drug misuse and relapse into drug use after withdrawal than men [27]. In ecstasy use, gender was found to significantly moderate the relationship between the consumption and design fluency [28]. When addicted to cocaine or amphetamine, women showed more impairment in decision making than men [29]. Studies exploring human sex-different responses to ketamine are rare. An animal study by Winters et al, who compared the differing analgesic effects of ketamine in male and female rats, indicated that females were much more sensitive to ketamine than males [30,31]. Moreover, female rats showed a greater sensitivity to ketamine neurotoxicity and brain neural loss compared to males [32]. Determining whether females are more susceptible than males to ketamine withdrawal symptoms and adverse effects is important, especially in cognitive deficits, because it associated with treatment retention and outcome in drug users [33]. The aim of this study is to further the management 
strategy for the current epidemic of ketamine abuse in Taiwan. The researchers collected information on relevant demographic and clinical data, so as to analyze the pattern of ketamine use, the discontinuation symptoms, the progression of harm, users' willingness to seek treatment, and gender differences among ketamine users in the Taipei metropolitan area.

\section{Methods}

\section{Participants and procedures}

In light of the increasingly severe problem of ketamine abuse, the Taiwanese juridical system implemented a new regulation that focused on ketamine users beginning in 2009. This new regulation stipulates that offenders caught by the police for using ketamine (as evidenced by urine screen results) will receive a fine ranging from 10,000 to $50,000 \mathrm{NT}$ dollars and attend a mandatory educational class for 4-8 hours. The mandatory class presents an opportunity for the investigator to come into contact with ketamine users within a larger sample.

We recruited ketamine offenders from February 2010 to May 2012 at the Kunming branch of the Taipei City Hospital where the educational classes are held. Since we were not aware of established questionnaires to examine ketamine-related problems, we designed a questionnaire to include demographic characteristics, age, reasons for initial use of ketamine, likes and dislikes during ketamine use, experience of discontinuation symptoms related to ketamine, and current concomitant substance use. The survey contained additional questions regarding the degree of memory impairment and urinary discomforts. Information about reasons for ketamine use and users' likes and dislikes during ketamine use was collected by open questions, while the remainder of the information was gathered through checklist questions. Since the Chinese version of the pelvic pain and urgency/frequency (PUF) questionnaire has been shown to be reliable and valid for assessment in patients with lower urinary tract symptoms associated with street-ketamine use [34], and the cut-off value of the total score range from 13-17 with detecting interstitial cystitis among different literature reviews $[34,35]$. We divided items assessing for urinary discomforts into three groups: Nil, Mild, and Severe, to separate the servility among the ketamine users who may beyond the cut-off points. Nil indicates never experiences of the pelvic pain or urgency/frequency; Mild indicates symptoms such as urgency/frequency occasionally but not usually; Severe indicates symptoms such as both pelvic pain and urgency/frequency, or one of the symptoms achieve usually or always, or having received clinical helps. The severe group could indicate the total score above 11 in the PUF questionnaire. However, there was no known validated self-report scale to evaluate ketamine-related memory impairments. Therefore, we designed a self-report questionnaire that categorizes memory impairment into three levels of severity: Nil, Mild, and Severe. Nil indicates no memory impairments; Mild indicates slight memory impairments; Severe indicates obvious signs of memory decline.

During the educational class, the research assistant provided a comprehensive description of the questionnaire, and all subjects were informed that their legal status would not be influenced by whether or not they participated in the study. The procedure was confidential. Participants were not required to identify themselves unless they wished to leave their personal information for referral to further medical evaluation or intervention. The Institutional Review Board of Taipei City Hospital, which has been certificated by Forum for Ethical Review Committees in Asian and Western Pacific Region (FERCAP) has approved this study (TCHIRB-971212).

\section{Data analysis}

Demographic and clinical characteristics of the patients were analyzed by Student $t$-test or Chi-square test. Statistical analyses were performed using SPSS version 12.0. Results were considered statistically significant at $\mathrm{p}<.05$. age-adjusted associations between genders and risk of Logistic regression analysis was also conducted to assess ketamine-related cognitive impairment or urinary symptoms.

\section{Results}

\section{Demographics}

Of the 1624 ketamine offenders, 10 either refused or failed to complete the questionnaire. Therefore, our analysis included data from 1614 participants (Mean age: $26.3 \pm 5.4$ years; Male to female ratio: 5:1). Demographic characteristics are displayed in Table 1, with gender comparison. Most of them were males (83.3\%), single (88.0\%) and employed (83.9\%). The average duration of ketamine use was about 4 years.

\section{Concomitant substance use and discontinuation symptoms}

Among the ketamine offenders, $72.4 \%$ smoked cigarettes and $16.5 \%$ consumed alcohol. It was found that more male than female users used betel nut concomitantly. Also, female users used more hypnotics and alcohol than males. The prevalence of other concomitant substance use among ketamine users is also displayed in Table 1.

When stopping ketamine use, the most common discomfort mentioned by participants was fatigue (26.0\%), followed by poor appetite (20.4\%) and drowsiness (19.1\%). Cravings, as well as anxiety and dysphoria, were common psychological symptoms at the discontinuation of ketamine use, especially in females. In addition, $3-5 \%$ of participants experienced physical symptoms such as palpitation, sweating or tremors during discontinuation. Gender differences 
Table 1 Demographics information and clinical data in ketamine users

\begin{tabular}{|c|c|c|c|c|c|}
\hline & & Total & Male & Female & $P$ value \\
\hline Age, years $\pm S D$ (range) & & $26.3 \pm 5.4(15-54)$ & $26.5 \pm 5.5(15-54)$ & $25.0 \pm 4.3(18-38)$ & $<0.001^{* * *}$ \\
\hline Gender, n (\%) & & 1614 & $1353(83.8 \%)$ & $261(16.2 \%)$ & $<0.001^{* * *}$ \\
\hline \multirow[t]{3}{*}{ Marriage status (\%) } & Single & 88.0 & 88.1 & 87.5 & $0.013^{*}$ \\
\hline & Married & 11.2 & 11.1 & 12.1 & \\
\hline & Others & 0.8 & 0.8 & 0.4 & \\
\hline Education, years $\pm S D$ & & $12.1 \pm 2.3$ & $12.2 \pm 2.4$ & $11.8 \pm 2.1$ & 0.056 \\
\hline \multirow[t]{2}{*}{ Occupation } & On job, n (\%) & $1354(83.9)$ & $1143(84.5)$ & $211(80.7)$ & \\
\hline & Jobless, n (\%) & $252(15.6)$ & $202(14.9)$ & $50(19.1)$ & \\
\hline Age of first ketamine use, years \pm SD & & $22.1 \pm 5.7$ & $22.3 \pm 5.8$ & $21.2 \pm 4.8$ & $0.001^{* *}$ \\
\hline \multirow[t]{9}{*}{ Other substance use (\%) } & Cigarette & 72.4 & 73.9 & 64.8 & $0.002^{* *}$ \\
\hline & Alcohol & 16.5 & 15.7 & 20.7 & $0.049^{*}$ \\
\hline & Betel nut & 15.8 & 18.4 & 2.3 & $<0.001^{* * *}$ \\
\hline & Hypnotics & 4.7 & 2.7 & 15.3 & $<0.001^{* * *}$ \\
\hline & MDMA & 4.4 & 4.1 & 5.7 & 0.246 \\
\hline & Meth-amphetamine & 1.6 & 1.4 & 2.7 & 0.133 \\
\hline & Marijuana & 1.5 & 1.6 & 1.1 & 0.622 \\
\hline & Heroin & 0.5 & 0.5 & 0.4 & 0.777 \\
\hline & Glue & 0.4 & 0.5 & 0.0 & 0.244 \\
\hline
\end{tabular}

$P$ value: difference between genders.

${ }^{*} \mathrm{p}<0.05,{ }^{* *} \mathrm{p}<0.01,{ }^{* * *} \mathrm{p}<0.001$.

in discontinuation symptoms are shown in Table 2. Female ketamine users presented with significantly more anxiety (23.4\%), dysphoria $(24.1 \%)$ and tremors $(6.5 \%)$ compared with male users during ketamine discontinuation.

\section{Self-report about the cognitive impairment, urinary} discomfort, and motivation to seek medical intervention $22.4 \%$ of total ketamine offenders reported that their cognitive ability was severely impaired by ketamine use, particularly in females (27.5\%). In addition, more than half of the total participants had urinary symptoms.
Compared with males, female ketamine offenders were more likely to develop severe cognitive impairment (OR, $95 \% \mathrm{CI}=1.717,1.075-2.741)$ and urinary symptoms $(2.719,1.501-4.928)$ according to their self-report. Gender differences in subjective reports of cognitive impairment and urinary tract symptoms among ketamine users are shown in Table 3.

Despite 76\% of ketamine users reporting cognitive impairment and $51.6 \%$ mentioning urinary symptoms, only $7 \%$ of ketamine users in our study opted to seek medical intervention or treatment under our transfer, with also

Table 2 Subjective reports of symptoms during ketamine discontinuation

\begin{tabular}{lllll}
\hline Discontinuation symptoms & Total (\%) & Male (\%) & Female (\%) & P-value \\
\hline Fatigue & 26.0 & 26.6 & 23.0 & 0.222 \\
Poor appetite & 20.4 & 20.7 & 18.8 & 0.481 \\
Drowsiness & 19.1 & 18.3 & 23.4 & 0.058 \\
Craving & 18.7 & 17.9 & 23.0 & 0.054 \\
Anxiety & 18.3 & 17.4 & 23.4 & $0.022^{*}$ \\
Dysphoria & 17.2 & 15.9 & 24.1 & $0.001^{* *}$ \\
Tremors & 3.6 & 3.0 & 6.5 & $0.006^{* *}$ \\
Palpitation & 5.0 & 4.5 & 7.3 & 0.059 \\
Sweating & 4.4 & 4.5 & 3.8 & 0.625 \\
Great effort to resist use & 4.0 & 3.9 & 4.6 & 0.609 \\
\hline
\end{tabular}

$P$ value: difference between genders.

${ }^{*} p<0.05,{ }^{* *} p<0.01$. 
Table 3 Subjective cognitive impairment and urinary tract symptoms among ketamine users and the adjusted odds ratios (AORs) between genders

\begin{tabular}{llllllll}
\hline & & Total & Male & Female & AORs $^{\mathbf{c}}$ & P value & $\mathbf{9 5 \%} \mathbf{C l}$ \\
\hline Cognitive impairment & Nil & $23.9 \%$ & $24.7 \%$ & $20.2 \%$ & reference & - & - \\
& mild & $53.6 \%$ & $53.9 \%$ & $52.3 \%$ & 1.275 & 0.258 & $0.837-1.942$ \\
& severe & $22.4 \%$ & $21.4 \%$ & $27.5 \%$ & 1.717 & $0.024^{*}$ & $1.075-2.741$ \\
Urinary tract symptoms & Nil & $49.5 \%$ & $49.7 \%$ & $48.4 \%$ & reference & - & - \\
& mild $^{a}$ & $45.8 \%$ & $46.5 \%$ & $41.9 \%$ & 0.915 & 0.601 & $0.656-1.276$ \\
& severe $^{b}$ & $4.8 \%$ & $3.8 \%$ & $9.7 \%$ & 2.719 & $0.001^{* *}$ & $1.501-4.928$ \\
\hline
\end{tabular}

andicating symptoms as urgency/frequency occasionally but not usually.

b Indicating symptoms as both pelvic pain and urgency/frequency, or one of the symptoms achieved usually or always, or having received clinical help.

'The reference category is Nil group in cognitive impairment and urinary tract symptoms respectively, after adjusted age.

$P$ value: the risk of subjective cognitive impairment or urinary tract symptoms between genders $95 \% \mathrm{Cl}, 95 \%$ confidence intervals.

${ }^{*} \mathrm{p}<0.05,{ }^{* *} \mathrm{p}<0.01$

statistically significant gender difference (male: 6.1\%, female:11.7\%; $\mathrm{p}=.001$ ).

\section{Discussion}

In this study, we explored the discontinuation symptoms of ketamine offenders in Taiwan, and examined the gender difference. The results demonstrated that female ketamine users had more severe self-report cognitive impairment and urinary symptoms related to ketamine use than male users. Our study is the first with a large sample size that showed a gender difference in the complications of ketamine use. The possible implication of our findings will be discussed.

\section{Discontinuation symptoms}

Pharmacologically, ketamine's main action is on glutamate, the major excitatory neurotransmitter in the brain. It is a non-competitive antagonist at one of the three glutamate receptors: the N-methyl d-aspartate (NMDA) receptor [3]. Ketamine also has less prominent actions at other receptor sites. It blocks muscarinic acetylcholine receptors and may potentiate the effects of gamma-aminobutyric acid (GABA) synaptic inhibition. In addition, ketamine induces activation of dopamine release [36] and acts as a weak agonist at $\mu$ opioid receptors [37]. Ketamine's affinity to multiple receptors could theoretically explain its effects on cognition, mood, psychotic experiences and withdrawal symptoms. Even though most of the participants in this study are polydrug users and the discontinuation symptoms reported here may have been the result of a combination of different substances, ketamine users related their symptoms to the discontinuation of ketamine in the questionnaire.

It is unclear if physical symptoms occur during ketamine withdrawal. However, the discontinuation symptoms mentioned in our questionnaire are quite similar to alcohol withdrawal. In fact, alcohol consumption was found to reduce certain ketamine discontinuation symptoms, such as anxiety, shaking, sweating, palpitation, and low mood [6]. Ketamine discontinuation can result in glutamate rebound with symptoms reminiscent of NMDA over-activity in alcohol withdrawal [6]. It is plausible that modified forms of alcohol withdrawal regimens can lessen symptoms of ketamine discontinuation.

\section{Gender difference}

Gender differences were found in the participants' subjective reports of discontinuation symptoms, as well as concomitant substance use and severity of impairment related to ketamine. In addition, female ketamine users reported a higher proportion of alcohol or hypnotic consumption compared to males. This tendency may be related to the significantly higher amounts of self-reported discontinuation symptoms such as anxiety and tremors in female ketamine users. In an animal study, it was suggested that the gonadal hormones in female rats played a critical role in enhancing the antidepressant-like effects of ketamine [38]. Therefore, we surmised that female ketamine users tended to experience dysphoric mood more frequently than males during ketamine discontinuation, as shown in our study.

A study about gender differences in abusers of amphetamine-type stimulants (ATS) and ketamine (K) in southwestern China [39] compared gender trends in ATS and ATS $+\mathrm{K}$ patients. For males, the ATS $+\mathrm{K}$ patients were more likely to develop psychotic disorders than ATS patients. For females, the ATS $+\mathrm{K}$ patients were more likely to develop cognitive impairment than ATS patients. Due to the central role of the NMDA-receptor in learning and memory, both acute and chronic use of ketamine exerts specific and wide-ranging effects on memory systems [4]. In line with previous animal study, female ketamine users were more vulnerable to cognitive deficits compared with males. On the other hand, a UK study showed an absence of gender difference in urinary symptoms among recreational ketamine users [40]. However, female ketamine offenders were more likely to develop severe urinary discomforts in our findings. This suggests that female ketamine users may experience a greater amount of 
impairment related to ketamine use, which may encourage them to seek medical intervention.

Biological mechanisms underlying female-specific ketamine effects are largely unknown. Acute administration of ketamine has been reported to decrease serum sex hormones including estradiol, progesterone, and testosterone in female rats [41]. It is possible that repeated ketamine may have specific effects on women via sex hormones; however, the probable cause of the gender differences found in this study requires further investigation.

\section{Limitation}

Our results should be interpreted with some limitations in mind. First, since participants were recruited while being mandated by the law to attend the class, it was difficult to validate the actual frequency and amount of ketamine and concomitant substance use, and thus the data related to substance use should be underestimated. We designed the confidential self-report to have favorable ascertainment. Second, in the absence of established questionnaires to examine ketamine-related problems (e.g., discontinuation symptoms), the questionnaires we used have not been studied to determine reliability and validity. There was no definition of cognitive impairment in the questionnaire, so the severity may be inconsistent between individual. Third, the discontinuation symptoms reported in our study may have been the result of a combination of different substances; however, ketamine users related their symptoms to the discontinuation of ketamine in the questionnaire. Researchers should be cautious to generalize our results given the high comorbidity of substance use disorder with other mental health issues. In this study, neuropsychiatric disorders or current affective state were not identified. Previous studies showed that the lifetime rates of mood and anxiety disorders are significantly higher among women than men, with and without substance-use disorders [42]. Associations between most substance use disorders and independent mood and anxiety disorders were positive and significant [43]. It is important to conduct a more comprehensive psychiatric assessment to determine whether substance use may enhance vulnerability for these disorders, or lead to organic changes that manifest as a mood or anxiety disorder. Finally, there might be gender differences on how participants responded to the selfreport questionnaires. Gender was an important demographic factor associated with symptom reporting, and most physical symptoms are typically reported more often by women than by men [44]. We cannot exclude the potential effects of gender on self-reporting biases in this study.

\section{Implications}

Ketamine has had a turbulent history since its first use as a clinical anesthesia for humans in 1964 [45]. With its analgesic-anesthetic mechanisms and antidepressant effect, ketamine is associated with a high potential for abuse or dependence. The pros and cons of ketamine use, as well as other areas of uncertainty regarding its use (e.g. antidepressant effect), deserve further studies in the future.

Despite several limitations generalizing data derived from our study, experience has shown us that investigating subjects with substance use can be extremely difficult. This is the first article demonstrating the correlation between gender and ketamine-related problems. Our study demonstrated that female ketamine users had more severe selfreported cognitive impairment and urinary symptoms than male users. These findings were compatible with previous animal study. However, a lot of additional work is required for a better understanding of gender differences in ketamine use. We hoped our study will stimulate further research in this field. Currently, there are no guidelines for effective management of ketamine withdrawal effects or dependence [46]. As ketamine abuse and dependence grows, addiction services need to be better informed of the effects of this drug, especially among young females. Policies emphasizing the increased vulnerability to ketaminerelated adverse effects in female users compared with male users and offering the opportunity to enhance medical accessibility may improve efforts to prevent progression of ketamine dependence. Further studies should aim to address the health problems experienced by this group and explore the best approach to treat ketamine addiction.

\section{Conclusions}

Discontinuation symptoms and physical side-effects are not uncommon in these ketamine offenders. Gender differences were noted in the subjective experience of discontinuation symptoms, concomitant substance use, and severity of impairment related to ketamine use. Additional work is required for a better understanding of the gender differences found in this study. Ketamine users in Taiwan are generally relatively young; therefore, further studies should be conducted to increase the availability and accessibility of effective treatment interventions, and preventative public education.

\begin{abstract}
Abbreviations
PCP: Phencyclidine; UNODC: United Nations Office on Drugs Control; NPS: New psychoactive substance; MDMA: 3,4-methylenedioxy-N-methylamphetamine;
\end{abstract} NMDA: N-methyl d-aspartate; GABA: Gamma-aminobutyric acid.

\section{Competing interests}

The authors declare that there is no conflict of interest of this study.

Authors' contributions

YWC and SKL designed the protocol and carried the survey. They gathered and analyzed the study's data, and wrote the first draft of the manuscript. $\mathrm{MCH}$ assisted with the study's design and in the preparation of this manuscript. All authors read and approved the final manuscript. 


\section{Acknowledgements}

This study was supported by Taipei City Government (99001-62-050) and granted from National Science Council, Taiwan (NSC 101-2314-B-532 -002 -MY2). The authors wish to express their deepest gratitude to all participants of this study.

Received: 27 December 2013 Accepted: 8 July 2014

Published: 22 September 2014

\section{References}

1. White PFWW, Trevor AJ: Ketamine-its pharmacology and therapeutic uses. Anesthesiology 1982, 56:119-136.

2. Berman RM, Cappiello A, Anand A, Oren DA, Heninger GR, Charney DS, Krystal JH: Antidepressant effects of ketamine in depressed patients. Biol Psychiatry 2000, 47(4):351-354.

3. Morgan CJ, Curran HV: Ketamine use: a review. Addiction 2011, 107(1):27-38.

4. Morgan CJ, Curran HV: Acute and chronic effects of ketamine upon human memory: a review. Psychopharmacology (Berl) 2006, 188(4):408-424.

5. Morgan CJ, Muetzelfeldt L, Curran HV: Consequences of chronic ketamine self-administration upon neurocognitive function and psychological wellbeing: a 1-year longitudinal study. Addiction 2010, 105(1):121-133.

6. Critchlow DG: A case of ketamine dependence with discontinuation symptoms. Addiction 2006, 101(8):1212-1213.

7. Lim DK: Ketamine associated psychedelic effects and dependence. Singapore Med J 2003, 44(1):31-34.

8. Blachut M, Solowiow K, Janus A, Ruman J, Cekus A, Matysiakiewicz J, Hese RT: [A case of ketamine dependence]. Psychiatr Pol 2009, 43(5):593-599.

9. Petersen RC SR, Stillman RC: Phencyclidine: an overview. NIDA Res Monogr $1978,21: 1-17$

10. Hurt PH, Ritchie EC: A case of ketamine dependence. Am J Psychiatry 1994, 151(5):779.

11. Jansen $K L$, rracotCankovic $R$ : The nonmedical use of ketamine, part two: $A$ review of problem use and dependence. J Psychoactive Drugs 2001, 33(2):151-158.

12. Morgan CJ, Monaghan L, Curran HV: Beyond the K-hole: a 3-year longitudinal investigation of the cognitive and subjective effects of ketamine in recreational users who have substantially reduced their use of the drug. Addiction 2004, 99(11):1450-1461.

13. Muetzelfeldt L, Kamboj SK, Rees H, Taylor J, Morgan CJ, Curran HV: Journey through the K-hole: phenomenological aspects of ketamine use. Drug Alcohol Depend 2008, 95(3):219-229.

14. Dillon P, Copeland J, Jansen K: Patterns of use and harms associated with non-medical ketamine use. Drug Alcohol Depend 2003, 69(1):23-28.

15. Cheng WC, Ng KM, Chan KK, Mok VK, Cheung BK: Roadside detection of impairment under the influence of ketamine-evaluation of ketamine impairment symptoms with reference to its concentration in oral fluid and urine. Forensic Sci Int 2007, 170(1):51-58.

16. Ng SH, Tse ML, Ng HW, Lau FL: Emergency department presentation of ketamine abusers in Hong Kong: a review of 233 cases. Hong Kong Med J 2010, 16(1):6-11.

17. Fang YX, Wang YB, Shi J, Liu ZM, Lu L: Recent trends in drug abuse in China. ActaPharmacol Sin 2006, 27(2):140-144.

18. The United Nations Office on Drugs and Crime (UNODC): World Drug Report. United Nations publication(Sales No. E.13.XI.6); 2013.

19. Chen WJ, Fu TC, Ting TT, Huang WL, Tang GM, Hsiao CK, Chen CY: Use of ecstasy and other psychoactive substances among school-attending adolescents in Taiwan: national surveys 2004-2006. BMC Public Health 2009, 9:27.

20. Lua AC, Lin HR, Tseng YT, Hu AR, Yeh PC: Profiles of urine samples from participants at rave party in Taiwan: prevalence of ketamine and MDMA abuse. Forensic Sci Int 2003, 136(1-3):47-51.

21. Yen CF, Hsu SY, Cheng CP: Polysubstance use and its correlates in adolescent ecstasy users in Taiwan. Addict Behav 2007, 32(10):2286-2291.

22. Leung KS, Li JH, Tsay WI, Callahan C, Liu SF, Hsu J, Hoffer L, Cottler LB: Dinosaur girls, candy girls, and Trinity: voices of Taiwanese club drug users. J Ethn Subst Abuse 2008, 7(3):237-257.

23. Tuchman E: Women and addiction: the importance of gender issues in substance abuse research. J Addict Dis 2010, 29(2):127-138.
24. Randall CL, Roberts JS, Del Boca FK, Carroll KM, Connors GJ, Mattson ME: Telescoping of landmark events associated with drinking: a gender comparison. J Stud Alcohol 1999, 60(2):252-260.

25. Bradley KA, Badrinath S, Bush K, Boyd-Wickizer J, Anawalt B: Medical risks for women who drink alcohol. J Gen Intern Med 1998, 13(9):627-639.

26. Anker JJ, Carroll ME: Females are more vulnerable to drug abuse than males: evidence from preclinical studies and the role of ovarian hormones. Curr Top Behav Neurosci 2011, 8:73-96.

27. Fattore $L$, Fadda P, Fratta W: Sex differences in the self-administration of cannabinoids and other drugs of abuse. Psychoneuroendocrinology 2009, 34(Suppl 1):S227-S236.

28. Medina KL, Shear PK, Corcoran K: Ecstasy (MDMA) exposure and neuropsychological functioning: a polydrug perspective. $J$ Int Neuropsychol Soc 2005, 11(6):753-765.

29. van der Plas EA, Crone EA, van den Wildenberg WP, Tranel D, Bechara A: Executive control deficits in substance-dependent individuals: a comparison of alcohol, cocaine, and methamphetamine and of men and women. J Clin Exp Neuropsychol 2009, 31(6):706-719.

30. Winters WD, Hance AJ, Cadd GC, Lakin ML: Seasonal and sex influences on ketamine-induced analgesia and catalepsy in the rat; a possible role for melatonin. Neuropharmacology 1986, 25(10):1095-1101.

31. Lees J, Hallak JE, Deakin JF, Dursun SM: Gender differences and the effects of ketamine in healthy volunteers. J Psychopharmacol 2004, 18(3):337-339.

32. Jevtovic-Todorovic V, Wozniak DF, Benshoff ND, Olney JW: A comparative evaluation of the neurotoxic properties of ketamine and nitrous oxide. Brain Res 2001, 895(1-2):264-267.

33. Sofuoglu M, DeVito EE, Waters AJ, Carroll KM: Cognitive enhancement as a treatment for drug addictions. Neuropharmacology 2013, 64:452-463.

34. Ng CM, Ma WK, To KC, Yiu MK: The Chinese version of the pelvic pain and urgency/frequency symptom scale: a useful assessment tool for street-ketamine abusers with lower urinary tract symptoms. Hong Kong Med J 2012, 18(2):123-130.

35. Kushner L, Moldwin RM: Efficiency of questionnaires used to screen for interstitial cystitis. J Urol 2006, 176(2):587-592.

36. Rabiner EA: Imaging of striatal dopamine release elicited with NMDA antagonists: is there anything there to be seen? I Psychopharmacol 2007, 21(3):253-258.

37. Oye I, Paulsen O, Maurset A: Effects of ketamine on sensory perception: evidence for a role of $\mathrm{N}$-methyl-D-aspartate receptors. $J$ Pharmacol Exp Ther 1992, 260(3):1209-1213.

38. Carrier N, Kabbaj M: Sex differences in the antidepressant-like effects of ketamine. Neuropharmacology 2013, 70:27-34.

39. Zhang Y, Lu C, Zhang J, Hu L, Song H, Li J, Kang L: Gender differences in abusers of amphetamine-type stimulants and ketamine in southwestern China. Addict Behav 2013, 38(1):1424-1430.

40. Winstock AR, Mitcheson L, Gillatt DA, Cottrell AM: The prevalence and natural history of urinary symptoms among recreational ketamine users. BJU Int 2012, 110(11):1762-1766.

41. Lee CJ, Do BR, Kim JK, Yoon YD: Pentobarbital and ketamine suppress serum concentrations of sex hormones in the female rat. $J$ Anesth 2000, 14(4):187-190

42. Greenfield SF, Back SE, Lawson K, Brady KT: Substance abuse in women. Psychiatr Clin North Am 2010, 33(2):339-355.

43. Grant BF, Stinson FS, Dawson DA, Chou SP, Dufour MC, Compton W, Pickering RP, Kaplan K: Prevalence and co-occurrence of substance use disorders and independentmood and anxiety disorders: Results from the national epidemiologic survey on alcohol and relatedconditions. Arch Gen Psychiatry 2004, 61(8):807-816.

44. Kroenke K, Spitzer RL: Gender differences in the reporting of physical and somatoform symptoms. Psychosom Med 1998, 60(2):150-155.

45. Domino EF: Taming the ketamine tiger. 1965. Anesthesiology 2010, 113(3):678-684.

46. Garg A, Sinha P, Kumar P, Prakash O: Use of naltrexone in ketamine dependence. Addict Behav 2014, 39(8):1215-1216.

\section{doi:10.1186/1747-597X-9-39}

Cite this article as: Chen et al:: Gender differences in subjective discontinuation symptoms associated with ketamine use. Substance Abuse Treatment, Prevention, and Policy 2014 9:39. 